\title{
La arquitectura de la empresa: Un elemento al servicio de la comunicación comercial
}

Francisco J. Caro González

A lo largo de la historia, la arquitectura ha sido empleada por los detentadores del poder como un reflejo simbólico del mismo. De esta forma, los faraones de Egipto construyeron grandes pirámides, en la Edad Media el poder eclesiástico se manifestó en la construcción de catedrales, la nobleza magnificaba su existencia con la edificación de palacios y los estados construían (y construyen) soberbios edificios para albergar sus instalaciones. En la actualidad, el cambio de valores hacia una sociedad cada vez más materialista hace que sea la empresa la que construya las catedrales de hoy, catedrales del dinero, que se convierten en hitos dentro de nuestras ciudades y que tratan de reflejar en muchos casos los valores y la función albergada en su interior. Los edificios de las empresas se insertan en el entramado urbano como un subsistema más, entre colegios, hospitales, viviendas, parques... De forma inevitable constituyen el paisaje que todos los ciudadanos disfrutamos o sufrimos cada día cuando vamos al trabajo o salimos a pasear. La arquitectura es uno de los elementos simbólicos más potentes que posee la empresa si se sabe utilizar de forma adecuada dentro de su estrategia de comunicación comercial.

En este artículo se resalta la importancia de la arquitectura de la empresa 
en la transmisión de su imagen. Tras centramos en la consideración de la empresa como institución y en el proceso de comunicación, estudiamos la arquitectura desde la perspectiva de la comunicación comercial, donde analizamos la relación entre los edificios y la publicidad, su contribución como reclamo comercial y el mecenazgo.

La concepción de la empresa como institución

La empresa se ha convertido en un actor fundamental de la sociedad actual. Su papel como elemento dinamizador la ha llevado a despojarse de la simple etiqueta de productora de bienes y servicios adquiriendo una mayor relevancia como ente social que no sólo cumple una función sino que además posee una conciencia, una filosofía y unos valores que la trascienden. La empresa se erige como una institución, ya no es una fábrica sino que se concibe además (adquiriendo conciencia) como el inspirador y el realizador de una misión dirigida al cuerpo social, como un miembro de la sociedad (Weil 1992).

Como ente social se inserta en un entorno con el que mantiene una relación de intercambio de flujos físicos e informativos. Los diferentes actores que conforman este entorno específico no se limitan a tener una actitud pasiva frente a ella. La mayor conciencia social y ecológica, el desarrollo de los medios de comunicación, las asociaciones de consumidores y las estrechas interrelaciones que se dan entre todos ellos, obligan a las empresas a mantener una actitud atenta y de colaboración con el ambiente en el que se desenvuelven. En este contexto la comunicación de la empresa no puede limitarse a una finalidad comercial, debe servir, además, como elemento de enlace que haga partícipes al resto de actores sociales de su proyecto, buscando su aprobación y adhesión. La organización empresarial se convierte en un agente social comprometido con su ambiente, al que debe respetar, favorecer e incluso dar ejemplo con una actuación ética y solidaria.

Pero no podemos dejar de lado el enfoque comercial de toda comunicación empresarial. La actual situación competitiva de muchos sectores hace indispensable que la empresa busque una diferenciación no sólo basada en 
la imagen de sus productos y servicios, sino en la de la propia empresa o institución, que muchas veces actúa en actividades diversas y precisa de una identidad global que permita su expansión y diversificación. Se pasa de la comunicación del producto o servicio a la comunicación de la marca, de la comunicación de la marca a la de la empresa y de la de la empresa a la de la institución o corporación. Norberto Chaves $(1988)^{1}$ en su enfoque comunicativo, indica que el solo acto de asumir la propia imagen pública como problemática y campo de intervenciones concretas es índice específico de una voluntad de institucionalización social.

La imagen de la empresa-institución

La empresa-institución es, por tanto, concebida como un sistema abierto y finalista que precisa mantener una fluida relación de intercambio con su entorno interno y externo para alcanzar sus objetivos.

La institución, como todo elemento social, posee una realidad objetiva que se manifiesta a través de sus actividades y discursos. La empresa capta de si misma una autoimagen, que no es más que una visión parcial y subjetiva de esa realidad. Esta realidad conforma su Identidad institucional, que se compone de atributos tanto positivos como negativos (Villafañe 1993: 43). El paso primordial en este proceso de comunicación es el conocimiento de la propia institución a través de una adecuada auditoría de imagen. Conocida lo mejor posible esta realidad, la empresa tratará de crear una identidad intencional frente a los públicos de su entomo, transmitiendo a través de múltiples soportes los atributos que según la misión, objetivos y estrategias empresariales sean más positivos. La percepción por parte de los actores de la empresa de la

1. Chaves (1988, págs:17-20) se decanta por el término «institución» frente a los conceptos «corporación» y «empresa». Nosotros utilizaremos en nuestro estudio de forma indistinta los términos «empresa» concebido de forma amplia y el de institución. También Pascal Weil (1992) considera que la empresa como miembro de la sociedad «se erige como institución». 
imagen intencional, y de la realidad de la empresa, le van a conformar una imagen pública que va a contribuir al éxito empresarial y social de la organización. La imagen se convierte en un elemento estratégico de primer orden, puesto que va a vehiculizar los valores y filosofía básicos de la empresa y va a representar una ventaja competitiva como elemento diferenciador.

\section{La imagen de las construcciones}

Para la transmisión de la imagen intencional de la institución la organización dispone de un complejo sistema comunicativo compuesto por dos grandes grupos de soportes. Los que son eminentemente gráficos y aquellos que hacen referencia a aspectos comportamentales de los propios miembros de la organización o grupos cercanos. Una posible tipología sería:

-Canales Personales: dentro de los cuales distinguimos entre canales controlables (los empleados, los dirigentes, los distribuidores) e incontrolables (prescriptores, líderes de opinión, la familia, los amigos)

-Canales Impersonales: también hacemos la distinción anterior entre canales controlables (el producto, los medios masivos y otras formas de comunicación comercial, los medios ambientales -dentro de los medios ambientales se consideran los diferentes aspectos exteriores e interiores de la empresa, que pueden servir para transmitir un mensaje determinado, entre ellos la arquitectura de sus edificios) e incontrolables (hechos causales y los medios masivos).

En la sociedad de la imagen los canales más visuales son los que ejercen una mayor influencia en la constitución de la imagen, ya que es la imagen visual el medio predominante tanto por su comprensión universal y por su ubicuidad como por la velocidad de lectura que permite (Pibernat 1989). Dentro de estos canales impersonales, controlables y con un gran impacto visual nos encontramos a nuestro objeto de reflexión, con la arquitectura de la empresa.

Si tratamos de ubicar la arquitectura de la empresa dentro de la teoría de la imagen, ésta se encuadraría en lo que Joan Costa (1987) denomina «dise- 
ño del medio ambiente» y que engloba, además del aspecto externo del edificio, al urbanismo y al interiorismo. Otros elementos que Costa considera para la construcción de la identidad de la empresa son «el diseño industrial», es decir los productos y «el diseño gráfico», donde inserta todo lo referente a la caligrafía, tipografía, ilustraciones y fotografías de las que hace uso la empresa. Es evidente la necesidad de interconexión y coherencia entre los distintos tipos de diseño, normalizados generalmente en los manuales de comunicación institucional.

\section{La arquitectura de la empresa}

Dentro del término arquitectura de la empresa englobamos a los edificios en los que se desarrollan sus múltiples actividades. Estas actividades son numerosas y con características muy diferentes. Por ello ofrecemos, en principio, una clasificación de los mismos en base a su funcionalidad:

-Edificios para el proceso productivo: almacenes, fábricas, laboratorios, centros de investigación, talleres, oficinas, sedes sociales.

-Edificios Comerciales (para la comercialización de productos o servicios): puntos de venta, puntos de atención al cliente.

-Edificios integrantes del servicio o producto: hoteles, parques de atracciones, etc.

De cada una de estos tipos de construcciones se podría hacer un estudio singularizado, nosotros emplearemos un enfoque generalista que iremos concretando en estudios próximos. No obstante, en este artículo, sólo consideraremos aquellos edificios «hechos a la medida», construidos de forma específica para esa empresa. Quedan al margen los edificios indiferenciados que acogen a diversas empresas.

La arquitectura como elemento de comunicación comercial

La comunicación en la empresa tradicionalmente ha estado encaminada a dar a conocer y estimular la venta de un producto o servicio, ¿puede la arquitectura contribuir a este objetivo básico? 
Es evidente que la respuesta variará dependiendo del tipo de edificio al que hagamos referencia, no sirve de igual manera un punto de venta que una sede social o una fábrica. Si retomamos la clasificación que apuntamos unas líneas más arriba, son los puntos de venta y los edificios integrantes del producto los que desempeñan con más naturalidad esta función. Dentro de este ámbito distinguimos dos posibilidades: el uso de la arquitectura de forma más puramente comercial, bajo los epígrafes arquitectura y publicidad, arquitectura reclamo; y una consideración más amplia que la comunicación exclusivamente comercial bajo los epígrafes arquitectura y mecenazgo y comunicación de valores.

\section{Arquitectura y publicidad}

1. La publicidad utiliza los medios de comunicación masivos para hacer llegar los mensajes al público objetivo. Dentro de estos medios masivos se encuentra la publicidad exterior. Los edificios de la empresa, gracias a su volumen, altura y ubicación pueden ser utilizados para sustentar los mensajes gráficos de la empresa. Dos casos representativos son los de COANBEGA en Sevilla y la enorme valla publicitaria de Coca Cola que exhibe en sus paredes y el de El Corte Inglés, que utiliza sus fachadas para las distintas campañas promocionales, «los ocho días», «las rebajas», «Oriente»...

2. En estrecha relación con la función simbólica de la arquitectura de la empresa, nos referiremos a los edificios como un componente del mensaje icónico de la publicidad. El edificio puede ser utilizada para codificar el contenido de los mensajes publicitarios. En las empresas de servicios, en las que no existe un producto que represente a la organización, es frecuente utilizar la imagen de sus sedes sociales para materializar la de la empresa. Es muy común en las entidades financieras y de seguros, que en muchos casos como explican Rodríguez, y Rodríguez (1995) han optado por la forma externa de sus edificios como ente de significación y a la vez como eje estratégico de sus campañas publicitarias. Este es el caso de Mapfre cuyo eslogan es edificios Mapfre, o las campañas televisivas de Santa Lucía cuyo plano final era la presentación ontológica y mayestática de su sede. 
Arquitectura reclamo

1. Son muchas las empresas que buscan espectacularidad y originalidad en el diseño de sus edificios para que sirvan como reclamo para los transeúntes y los invite a entrar en su interior. Esto ha sido llevado al extremo por algunos arquitectos como Venturi, Graves y Stern, representantes del Postmodernismo, que construyen fachadas atrayentes, que se derrumban, se despegan o cuartean, mostrando un caos totalmente controlado. La forma exterior no guarda ninguna relación con la normalidad del interior del edificio. Las construcciones pretenden sorprender al público alcanzando una fuerte notoriedad que perdura en la memoria (Capote Abreu 1994). Son buenos ejemplos el de los almacenes BEST en Sacramento (EE.UU.) de SITE Project y el edificio de oficinas de la agencia de publicidad Chiat/Day/Mojo diseñado por Gehry en Venice (California), constituido por unos enormes prismáticos bajo cuyos binoculares transcurre el tráfico rodado al edificio.

2. Los edificios soportan las enseñas de la empresa para facilitar su reconocimiento por parte de los clientes. Sobre sus fachadas, mejor o peor integrados, podemos encontrar los diferentes elementos visuales que constituyen la simbología de la empresa: anagrama, logotipo, los colores....

\section{Arquitectura y mecenazgo}

La Arquitectura es un arte. A parte de su valor funcional posee un valor estético y cultural que viene determinado por los movimientos artísticos de cada época y por las tecnologías en uso.

Un buen edificio permite al empresario aprovechar las ventajas de la popularidad que su difusión como obra de arte le puede reportar. El efecto comunicador de esta modalidad se multiplica hoy día gracias al creciente interés de los medios de comunicación por la arquitectura, los periódicos y revistas de contenido generalista ${ }^{2}$ incorporan secciones sobre esta discipli-

2. El diario El País, en su suplemento cultural Babelia tiene una sección de Arquitectura; concretamente el sábado 25 de noviembre de 1995, dedica su portada 
na, y en las ciudades se publican guías de arquitectura en las que cada vez se añaden más edificios contemporáneos, entre los cuales existe una importante presencia de los edificios de las empresas. Sirva de ejemplo la Guía de Arquitectura de Sevilla y Área Metropolitana siglo XX en la que el $45 \%$ de los edificios recomendados son de carácter industrial o comercial.

Ya a principios del siglo XX en la revista alemana Der Industriebau se comentaba acerca de la fábrica de hormas de zapatos Fagus de W. Gropius que muchos fabricantes admiten que una colaboración artística en la construcción de las instalaciones puede producir algo de lo que la industria actual no puede prescindir: una publicidad del más noble rango (Capote Abreu 1994).

La arquitectura no sólo se concibe como un instrumento indispensable para el desarrollo de la actividad productiva, o un escaparate para impresionar o atraer a los clientes, sino que en tanto que arte puede ser utilizada en la estrategia de comunicación de la empresa dando a conocer su obra. Por ello las grandes firmas acuden a arquitectos reputados que ofrecen una garantía profesional y facilitan los mensajes promocionales y publicitarios, lo que hace que su arquitectura sea una inversión rentable. A Herbert Johnson le gustaba recordar que el edificio que Wright le había construido le había ahorrado millones de dólares en publicidad (Ferrier 1989). De igual manera el industrial alemán Braun mantiene que una fábrica construida en un estilo puramente utilitario le habría costado tal vez un 3\% menos, y que merece la pena pagar la pequeña cantidad suplementaria por la fama que este diseño dará a su empresa, independientemente de que tal vez haya financiado una obra de arte (Maxwell 1992)

Grandes empresas como Disney y Vitra están siendo para la arquitectura reciente lo que fue $A E G$ en las primeras décadas del siglo y Olivetti en el

\footnotetext{
y dos páginas interiores al arquitecto Richard Meier (Autor de la sede de Canal + en Francia). El País Semanal del domingo 26 de noviembre dedica 6 páginas a una entrevista con Norman Foster, y 4 páginas más a 10 obras emblemáticas de la arquitectura de los últimos diez años, en las que aparece en primer lugar el Banco de Hong Kong y Shangai de Norman Foster.
} 
periodo de entreguerras: unas compañías que se han convertido en la mayores patrocinadoras de arquitectura de autor (Fernández-Galiano 1994) y para las que trabajan los arquitectos con más renombre del momento como Graves, Moore, Stern, Predock, Isozaki, Gehry, Siza, etc.

\section{Comunicación de valores}

Hemos comentado varias veces que los edificios de la empresa son una de las partes más visibles e inmutables de la misma y cómo en muchos casos su sedentarismo se ve roto por la posibilidad de hacer viajar su imagen a través de los medios de comunicación, bien sea gracias a la publicidad o a la publicity que puede proporcionar el mecenazgo. Al igual que las personas hacen alarde de su riqueza habitando una lujosa vivienda, las empresas tratan de reflejar su éxito en las fachadas de sus edificios. Transmiten a través de ellas riqueza y poder al entorno que las rodea. Un claro ejemplo es el Banco de Inglaterra (1790-1832) obra de Sir John Soane que tratan de reflejar la suntuosidad de la empresa y dar una imagen de seguridad, estabilidad y confianza a los clientes y la sociedad en general a través de un edifico majestuoso. En un comentario de Tellería (1991) sobre los edificios bancarios encontramos: «Es una arquitectura que se caracteriza por un aparente derroche de espacio y los materiales y decoración se correspondían con la consideración de que la categoría de una empresa debía reflejarse en la monumentalidad de su sede, que además formaba parte valiosa de su patrimonio». Un ejemplo actual podría ser el edifico de los arquitectos Lluís Clotet \& Ignacio Paricio para el Banco de España en Gerona (1991) en el que se ha buscado la monumentalidad exterior que simbolice el prestigio de esta organización.

Además de éxito empresarial, las empresas a través de sus edificios pueden transmitir valores como transparencia, equilibrio, armonía, humanidad. También valores más comerciales como innovación, modernidad, tecnología, sofisticación... que contribuyen al posicionamiento estratégico de la empresa. Veamos dos ejemplos tomados de la Exposición Universal de Sevilla.

La empresa Siemens construyó para la Expo 92 de Sevilla un edificio que debía representar a la empresa por sus contenidos expositivos y también 
por su propia concepción arquitectónica. Claridad estructural, simplicidad y precisión tecnológica son algunos de los mensajes transmitidos a partir de la utilización de un mensaje arquitectónico de fácil lectura. En la actualidad este edificio es un centro de desarrollo tecnológico (VV. AA. 1992).

Otro edificio representativo es el pabellón de la ONCE. Los arquitectos sólo tenían una idea básica como punto de partida, la filosofía de la institución promotora: integración y accesibilidad. Frente a los binomios cegueraoscuridad, minusvalía-ocultación, el edificio se abre al exterior, se desnuda, se hace transparente, se funde con la Cartuja, deja que la luz y los reflejos del resto de la Exposición penetren por su piel. Es la ocasión de contemplar y ser contemplado, participar e invitar a la participación (idem).

En muchos casos, los edificios de las empresas reflejan la filosofía básica de la empresa. Eisner, Presidente de Disney persigue crear «arquitectura para la diversión» en sus parques de atracciones. Se busca una estética, que sin ser determinada de antemano, sí se encamina al deleite del usuario y connota diversión, espectáculo, al igual que lo hace la marca Disney.

El carácter estratégico de la arquitectura de la empresa

Los distintos aspectos comunicativos de la arquitectura de la empresa nos muestran la importancia de este elemento a la hora de desarrollar una estrategia de comunicación y definir la imagen pública de la organización. Este carácter es aun más patente si consideramos que la vida media de la estructura externa de un edificio se calcula en unos 40 ó 50 años (Duffy 1980) y que la inversión necesaria para adquirir o construir un edificio es de las más elevadas que debe realizar la empresa. Como elemento comunicativo debe constituir un todo integrado en la estrategia de comunicación con el resto de los soportes. Por todo esto es necesario que el empresario en el momento de realizar esta inversión tenga muy claras la misión y estrategia de la empresa y trate de encontrar un diseño arquitectónico que responda a esos parámetros fundamentales. Los edificios de las empresas tienen que adaptarse a las necesidades presentes y futuras del entorno en que se desenvuelve y considerar las capacidades específicas de la organización. También debe tenerse 
en cuenta, que la arquitectura, como todo movimiento relacionado con la imagen se ve afectada por la moda, lo que supone que la imagen que se quiera transmitir debe ser muy bien meditada y reflejar la visión a muy largo plazo de la empresa. Frente a esto no podemos olvidar la máxima de que «la buena arquitectura no pasa de moda».

Al igual que en las campañas publicitarias es deseable una estrecha relación anunciante-agencia, el empresario tiene que proporcionar al arquitecto las claves fundamentales de los edificios de la empresa, no sólo respecto a aspectos funcionales, sino también estéticos.

Hacia una arquitectura de la empresa

A modo de conclusión presentamos algunas consideraciones básicas que el empresario debe analizar en el momento de plantearse la construcción o compra de un edificio para su empresa.

- La arquitectura de la empresa puede jugar un papel fundamental en la comunicación comercial, sobre todo en las de servicios donde no existe un producto físico con el que asociar a la empresa.

- Los edificios empresariales se constituyen como potentes soportes para vehicular la identidad de la empresa hacia el exterior de la misma. Es fundamental que se guarde una coherencia entre la simbología de los edificios y el resto de los soportes comunicativos de la institución.

-La inversión en un edificio fabril o una sede social debe ser contemplada como una inversión en patrimonio, mientras más relevante sea, y mientras mayor sea su valor cultural y su calidad, más grandes serán también los beneficios reportados por el fondo de comercio creado o por el prestigio que supone el mecenazgo de una obra de arte y la difusión de la misma.

-La imagen es necesaria, pero no podemos olvidar que ante todo el edificio constituye la estructura para el funcionamiento eficaz de la empresa. Estética y funcionalidad no están reñidas. 


\section{REFERENCIAS BIBLIOGRÁFICAS}

VV. AA. (1992): «La arquitectura de la Expo 92», en ON Diseño, №. monográfico. CAPOTE ABREU, J. (1994): Construcción y edificación industrial, Santander, Servicio de Publicaciones de la E.T.S. de Ingenieros de Caminos, Canales y Puertos de Santander.

CHAVES, N. (1988): La imagen corporativa, Barcelona, Gustavo Gili.

COSTA, J. (1987): Imagen global, Barcelona, Ediciones CEAC.

DUFFY, F. et al. (1980): Oficinas, Blume.

FERNÁNDEZ-GALIANO, L. (1994): «1993, doce meses del mundo», en A\&V $n^{\circ} 45-46$.

FERRIER, J. (1989): Usines, Paris, Editions du Moniteur.

FISHER, T. (1991): «El tiempo y su medida. Isozaki en Disney World», en Arquitectura Viva, $\mathrm{n}^{\circ} 21$.

MAXWELL, R. (1992): «El último Stirling», en Arquitectura Viva, nº 27.

PHII LIPS, A. (1993): Arquitectura Industrial, Barcelona, Gustavo Gili.

PIBERNAT \& DOMÈNECH, O. (1989): «La gestión institucional de la imagen», en Diseño e Imagen Corporativa en las Instituciones Públicas, Madrid, IMPI.

RODRIGUEZ, J. C. \& RODRIGUEZ, C. (1995): «La arquitectura como elemento de formación de la imagen corporativa», en J. REY (ed.): Algunas consideraciones sobre la comunicación empresarial e institucional, Sevilla, Questiones Publicitarias /Número monográfico.

TELLERÍA, A. (1991): «La arquitectura del dinero», en Oficinas de concepto innovador, Diseño Interior/Número Monográfico.

VILLAFAÑE, J. (1993): Imagen Positiva, Madrid, Pirámide.

WEIL, P. (1992): La comunicación global, Barcelona, Paidós. 